\title{
CONNECTEDNESS OF HIGGS BUNDLE MODULI FOR COMPLEX REDUCTIVE LIE GROUPS
}

\author{
OSCAR GARCÍA-PRADA AND ANDRÉ OLIVEIRA
}

\begin{abstract}
We carry an intrinsic approach to the study of the connectedness of the moduli space $\mathcal{M}_{G}$ of $G$-Higgs bundles, over a compact Riemann surface, when $G$ is a complex reductive (not necessarily connected) Lie group. We prove that the number of connected components of $\mathcal{M}_{G}$ is indexed by the corresponding topological invariants. In particular, this gives an alternative proof of the counting by J. Li in 25] of the number of connected components of the moduli space of flat $G$-connections in the case in which $G$ is connected and semisimple.
\end{abstract}

\section{INTRODUCTION}

The topology of the moduli spaces $\mathcal{M}_{G}$ of $G$-Higgs bundles over a compact Riemann surface $X$ (of genus $g \geqslant 2$ ) has been object of intense study in the past decade, mostly by making use of the Morse theoretic techniques introduced by Nigel Hitchin in the seminal paper [22] on Higgs bundles. This procedure uses the fact that the moduli spaces $\mathcal{M}_{G}$ carry a proper, bounded below real function $f$, from which we can obtain information at least about the connected components, through the study of the subvarieties of local minima of $f$. In some good cases, namely when the spaces $\mathcal{M}_{G}$ are smooth, the Poincaré polynomial may be calculated, by a study of all critical subvarieties of $f$, since in these cases $f$ is indeed a perfect Morse-Bott function.

The connected components of $\mathcal{M}_{G}$ have been object of investigation for many families of real reductive Lie groups $G$, especially after the work of Hitchin [23] where the case $G=\operatorname{SL}(n, \mathbb{R})$ was addressed; some references where this subject was studied are [1, 6, 17, 8, 10, 15, 16, 17, 18, 20, 21, 28]. The approach has been through a case-by-case study concerning the classes of $G$. So, the aim of this paper is to take a first step towards the computation of the number of connected components of $\mathcal{M}_{G}$, from an intrinsic point of view, in the sense that we do not specify the group $G$. It is an abstract approach using the above mentioned techniques introduced by Hitchin in $[22$ for $G=\mathrm{SL}(2, \mathbb{C})$. Let $c$ represent a topological class of $G$-Higgs bundles and let $\mathcal{M}_{G}(c)$ denote the subspace of $\mathcal{M}_{G}$ whose points represent those $G$-Higgs

Date: 17 March 2016.

2010 Mathematics Subject Classification. 14D20, 14F45, 14H60.

Key words and phrases. Semistable Higgs bundles, connected components of moduli spaces.

Members of GEAR (Geometric Structures and Representation Varieties). First author partially supported by the Ministerio de Economía y Competitividad of Spain through Project MTM2010-17717 and Severo Ochoa Excellence Grant. Second author partially supported by CMUP (UID/MAT/00144/2013) and CM-UTAD (PEst-OE/MAT/UI4080/2014), by the Projects PTDC/MAT/120411/2010, PTDC/MAT-GEO/0675/2012 and EXCL/MAT-GEO/0222/2012 and finally by the Post-Doctoral fellowship SFRH/BPD/100996/2014. All these are funded by FCT (Portugal) with national (MEC) and European structural funds (FEDER), under the partnership agreement PT2020. The second author also thanks the Instituto de Ciencias Matemáticas (ICMAT) in Madrid and the Institute for Mathematical Sciences (IMS) in Singapore — that visited while preparing the paper - for the excellent conditions provided.

The authors thank Peter Dalakov for useful comments to a preliminary version of the paper and also Tony Pantev for useful discussions concerning the paper [12]. 
bundles within the class $c$. Of course, $\mathcal{M}_{G}(c)$ is a union of connected components. We consider a general complex reductive Lie group and we prove the following (see Theorem 5.3).

Theorem 1.1. For any class $c$, the moduli space $\mathcal{M}_{G}(c)$ is non-empty and connected for any complex reductive Lie group $G$.

Some particular cases of this theorem have already been proved in [25, 12, 24] — see Remark 5.4 - but all of them use different methods from the ones we use in this paper.

Recall that $\pi_{1} X$ is a finitely generated group, with $2 g$ generators $a_{1}, b_{1}, \ldots, a_{g}, b_{g}$ such that the product of all commutators $\left[a_{i}, b_{i}\right]$ is trivial. Now, let $\Gamma$ be the universal central extension of $\pi_{1} X$, defined as the finitely generated group, with $2 g+1$ generators $a_{1}, b_{1}, \ldots, a_{g}, b_{g}, \delta$, such that $\delta$ lies in the center of $\Gamma$ and $\prod_{i=1}^{g}\left[a_{i}, b_{i}\right]=\delta$. Define $\Gamma_{\mathbb{R}}$ as $\mathbb{R} \times_{\mathbb{Z}} \Gamma$, where $\mathbb{Z}$ is identified as the subgroup of $\Gamma$ generated by $\delta$. Given any real reductive Lie group $G$, a reductive representation of $\Gamma_{\mathbb{R}}$ in $G$ is a continuous homomorphism $\rho: \Gamma_{\mathbb{R}} \rightarrow G$ which becomes completely reducible when composed with the adjoint representation of $G$. A reductive representation $\rho$ is said to be central if $\rho(\mathbb{R})$ lies in the centre of $G_{0}$, the identity component of $G$. Let Hom $_{\text {cent }}^{\text {red }}\left(\Gamma_{\mathbb{R}}, G\right)$ be the space of such reductive, central representations. The group $G$ acts on this space by conjugation and we denote by $\mathcal{R}_{G}$ the quotient space, usually called the $G$-character variety of $X$.

For any real reductive Lie group $G$, non-abelian Hodge theory provides a homeomorphism between $\mathcal{M}_{G}$ and $\mathcal{R}_{G}$, so our result shows that $\mathcal{R}_{G}(c)$ is connected for any complex reductive Lie group and for any class $c$. Here $\mathcal{R}_{G}(c)$ is the subspace of $\mathcal{R}_{G}$ whose corresponding central curvature principal $G$-bundle lies in the topological class $c$ (the homeomorphism mentioned above respects the topological classes). If $G$ is complex, connected and semisimple, then $c$ is trivial and one consider representations of $\pi_{1} X$ in $G$. In this case, the connectedness of $\mathcal{R}_{G}$ is known for more than twenty years, by the work [25] of Jun Li. Hence, the corresponding result on the side of Higgs bundles also follows. However, Li's methods do not use Higgs bundles. A Higgs bundle approach for $G=\mathrm{SL}(2, \mathbb{C})$ was given by Hitchin in 22 and for $G=\mathrm{GL}(n, \mathbb{C})$ by Simpson [36]. In this paper we give an alternative proof to the result of Jun Li using Higgs bundles. Moreover, our result is more general in the sense that it is valid for reductive and even non-connected complex Lie groups. Along the way, we prove other results about bundles which we have not been able to find in the literature. We highlight the following theorem which describes the stable and non-simple Higgs bundles, which give rise to orbifold type singularities of the moduli space $\mathcal{M}_{G}$. As far as we know, this result does not appear in the literature even for principal bundles, and our proof also holds in that case (see Theorem 3.16).

Theorem 1.2. Let $G$ be a complex Lie group. For any stable and non-simple G-Higgs bundle, there is a complex reductive Lie subgroup $G^{\prime} \subset G$ such that the $G$-Higgs bundle admits a reduction of structure group to $G^{\prime}$ and it is stable and simple as a $G^{\prime}$-Higgs bundle.

A natural generalization of this work is to consider a general real reductive Lie group and we intend to pursue this direction in a different paper. In this case, it is well-known that Theorem 1.1 does not hold, as there are some classes of real groups for which $\mathcal{M}_{G}$ has "extra" components.

\section{2. $G$-Higgs BUndLES AND tOpological InVARIANTS}

In this section we introduce the main objects which we shall work with. These are called G-Higgs bundles and roughly are pairs consisting of a holomorphic bundle and a section of an associated bundle. G-Higgs bundles can be defined on any compact Kähler manifold (cf. 
[35]), and $G$ may be any real reductive Lie group (see for example [8]), but we will restrict ourselves to $G$-Higgs bundles over compact Riemann surfaces, and such that $G$ is a complex reductive Lie group.

Fix a compact and connected Riemann surface $X$ of genus $g \geqslant 2$. Let $K=T^{*} X^{1,0}$ be its canonical line bundle. Let $G$ be a complex reductive Lie group. Given a principal $G$-bundle $E_{G}$, denote by $\operatorname{ad}\left(E_{G}\right)$ the adjoint bundle of $E_{G}$, that is the vector bundle obtained from $E_{G}$ under the adjoint representation of $G$ on its Lie algebra $\mathfrak{g}$ :

$$
\operatorname{ad}\left(E_{G}\right)=E_{G} \times_{G} \mathfrak{g} .
$$

Definition 2.1. A $G$-Higgs bundle over $X$ is a pair $\left(E_{G}, \varphi\right)$ where $E_{G}$ is a holomorphic principal $G$-bundle over $X$ and $\varphi$ is a holomorphic section of $\operatorname{ad}\left(E_{G}\right) \otimes K$. The section $\varphi$ is usually called the Higgs field.

As an example, a $\mathrm{GL}(n, \mathbb{C})$-Higgs bundle or simply a Higgs bundle is, in terms of holomorphic vector bundles, a pair $(V, \varphi)$ with $V$ a holomorphic rank $n$ vector bundle and $\varphi \in H^{0}(X, \operatorname{End}(V) \otimes K)$, whereas for $\mathrm{SL}(n, \mathbb{C})$-Higgs bundles, $V$ is required to have trivial determinant and $\varphi$ must be trace-free. Higgs bundles were first introduced by Hitchin in 22 , for $G=\operatorname{SL}(2, \mathbb{C})$, while studying the self-duality equations (now known as Hitchin equations) on Riemann surfaces.

The topological class of a $G$-Higgs bundle is given by the topological class of the underlying $G$-principal bundle. If $G$ is connected, the topological classification of principal $G$-bundles over the compact Riemann surface $X$ is well-known to be given by elements of $\pi_{1} G$ (cf. [30, Proposition 5.1]). For a not necessarily connected group $G$, the topological classification of G-bundles is more subtle, and we only briefly sketch it; details can be found in [27, §2] and in [28, Prop. 3.1]. First we assume that $\pi_{0} G$ is an abelian group; this assumption is only needed for Theorem 2.2 below and nothing else. Given a principal $G$-bundle $E_{G}$ on $X$, let $m_{1}\left(E_{G}\right)$ be the induced (flat) $\pi_{0} G$-bundle. This gives a first topological invariant of $E_{G}$, as

$$
m_{1}\left(E_{G}\right) \in H^{1}\left(X, \pi_{0} G\right) \cong\left(\pi_{0} G\right)^{2 g} .
$$

Now, $\pi_{0} G$ acts on $\pi_{1} G$ through the conjugation action of $G$ on itself. Fix a class $m_{1} \in$ $H^{1}\left(X, \pi_{0} G\right)$ and let $\pi_{1} \mathcal{G}_{m_{1}}$ be the flat $\pi_{1} G$-bundle associated to $m_{1}: \pi_{1} X \rightarrow \pi_{0} G$ via the action $\pi_{0} G \rightarrow \operatorname{Aut}\left(\pi_{1} G\right)$. We can consider cohomology with values in the local coefficient system $\pi_{1} \mathcal{G}_{m_{1}}$. In fact, $\pi_{0} G$ also acts on $H^{2}\left(X, \pi_{1} \mathcal{G}_{m_{1}}\right)$ through $\pi_{0} G \rightarrow \operatorname{Aut}\left(\pi_{1} G\right)$, and the next result says that topological $G$-bundles on $X$ with the first class $m_{1}$ fixed are classified by elements in the quotient space.

Theorem 2.2 (28, Proposition 3.1). Let $G$ be a Lie group with $\pi_{0} G$ abelian. There is a bijection between the set of isomorphism classes of continuous principal $G$-bundles over the surface $X$ with invariant $m_{1} \in\left(\pi_{0} G\right)^{2 g}$ and the quotient set $H^{2}\left(X, \pi_{1} \mathcal{G}_{m_{1}}\right) / \pi_{0} G$.

In fact, this theorem is valid not only on surfaces, but on any 2-dimensional connected $C W$-complex. Observe that if $G$ is connected, the preceding theorem gives the bijection between topological classes of $G$-Higgs bundles over $X$ and $H^{2}\left(X, \pi_{1} G\right) \cong \pi_{1} G$, as we already mentioned.

\section{Semistability AND MOduli SPACES}

3.1. Semistability. Let $G$ be a complex reductive Lie group. In order to consider moduli spaces of $G$-Higgs bundles we need the corresponding notions of (semi,poly)stability. We briefly recall the main definitions. The main reference is [14, where all these general notions are deduced in detail. 
We recall first some definitions. Let $\mathfrak{g}$ be the Lie algebra of $G$ and $\mathfrak{z}$ its centre. Then $\mathfrak{g}=\mathfrak{z} \oplus \mathfrak{g}_{s s}$, where $\mathfrak{g}_{s s}=[\mathfrak{g}, \mathfrak{g}]$ is the semisimple part of $\mathfrak{g}$. Given a Cartan subalgebra $\mathfrak{c}$ of $\mathfrak{g}_{s s}$, we will consider roots of $\mathfrak{g}$ as forms on $\mathfrak{c}$ extended by zero on $\mathfrak{z}$. Let $R$ be the set of such roots and for $\alpha \in R$, let $\mathfrak{g}_{\alpha}$ be the corresponding root space, so that we have the corresponding decomposition:

$$
\mathfrak{g}=\mathfrak{z} \oplus \mathfrak{c} \oplus \bigoplus_{\alpha \in R} \mathfrak{g}_{\alpha}
$$

Let $\Delta \subset R$ be the system of simple roots.

Let $\mathfrak{h}$ be the Lie algebra of a maximal compact subgroup of $G$. Given $s \in i \mathfrak{h}$,

$$
P_{s}=\left\{g \in G \mid e^{t s} g e^{-t s} \text { remains bounded as } t \rightarrow \infty\right\},
$$

is a parabolic subgroup of $G$, whose corresponding parabolic subalgebra of $\mathfrak{g}$ is

$$
\mathfrak{p}_{s}=\left\{v \in \mathfrak{g} \mid \operatorname{Ad}\left(e^{t s}\right)(v) \text { remains bounded as } t \rightarrow \infty\right\}
$$

If, moreover, we define

$$
L_{s}=\left\{g \in G \mid \lim _{t \rightarrow \infty} e^{t s} g e^{-t s}=g\right\}
$$

then $L_{s} \subset P_{s}$ is a Levi subgroup of $P_{s}$, and

$$
\mathfrak{l}_{s}=\left\{v \in \mathfrak{g} \mid \lim _{t \rightarrow \infty} \operatorname{Ad}\left(e^{t s}\right)(v)=0\right\}
$$

is the corresponding Levi subalgebra of $\mathfrak{p}_{s}$.

In case $G$ is connected, every parabolic subgroup $P$ is of the form (3.1) for some $s \in i \mathfrak{h}$; the same holds for the Levi subgroups. For $G$ non-connected that may not be the case (cf. [26, Remark 5.3]). However, in order to define semistability, the parabolic subgroups which need to be considered are precisely the ones of the form (3.1). Hence, for simplicity, and when no explicit mention to $s \in i \mathfrak{h}$ is needed, we refer to these as the parabolic subgroups of $G$, keeping in mind that we mean the groups defined by (3.1). We will do the same for the Levi subgroups, referring to (3.2).

Let then $P$ be a parabolic subgroup of $G$. A character of the Lie algebra $\mathfrak{p}$ of $P$ is a complex linear map $\mathfrak{p} \rightarrow \mathbb{C}$ which factors through $\mathfrak{p} /[\mathfrak{p}, \mathfrak{p}]$. Let $\mathfrak{l} \subset \mathfrak{p}$ be the corresponding Levi subalgebra and let $\mathfrak{z} \mathfrak{l}$ be the centre of $\mathfrak{l}$. Then, one has that $(\mathfrak{p} /[\mathfrak{p}, \mathfrak{p}])^{*} \cong \mathfrak{z}_{\mathfrak{l}}^{*}$, so the characters of $\mathfrak{p}$ are indeed classified by elements of $\mathfrak{z}_{\mathfrak{l}}$. Since $\mathfrak{g}$ is reductive, the Killing form on its semisimple part extends to a non-degenerate invariant $\mathbb{C}$-bilinear pairing $\langle\cdot, \cdot\rangle$ on $\mathfrak{g}$, which yields an isomorphism $\mathfrak{z}_{\mathfrak{l}}^{*} \cong \mathfrak{z} \mathfrak{l}$. Thus, a character $\chi_{*} \in \mathfrak{z}_{\mathfrak{l}}^{*}$ of $\mathfrak{p}$ is uniquely determined by an element $s_{\chi_{*}} \in \mathfrak{z} \mathfrak{l}$. Indeed, it can be shown that $\mathfrak{z} \mathfrak{l} \subset i \mathfrak{h}$, so that $s_{\chi_{*}} \in i \mathfrak{h}$. Now, the character $\chi_{*}$ of $\mathfrak{p}$ is said to be antidominant if $\mathfrak{p} \subset \mathfrak{p}_{s_{\chi_{*}}}$ and strictly antidominant if $\mathfrak{p}=\mathfrak{p}_{s_{\chi_{*}}}$.

Given a character $\chi: P \rightarrow \mathbb{C}^{*}$ of $P$, denote by $\chi_{*}$ the corresponding character of $\mathfrak{p}$. We say that $\chi$ is (strictly) antidominant if $\chi_{*}$ is.

Let $E_{G}$ be a holomorphic principal $G$-bundle on $X$ and let $P$ be a parabolic subgroup of $G$. Denote by $E_{G}(G / P)$ the holomorphic bundle with fibre $G / P$ associated to $E_{G}$ and to the standard action of $G$ on $G / P$. The bundle $E_{G}(G / P)$ is canonically isomorphic $E_{G} / P$. Let $\sigma \in H^{0}\left(X, E_{G} / P\right)$, that is, a reduction of the structure group of $E_{G}$ to $P$, and denote by $E_{\sigma} \subset E_{G}$ the corresponding holomorphic principal $P$-bundle on $X$. So, $E_{\sigma}$ is the pullback of the principal $P$-bundle $E_{G} \rightarrow E_{G} / P$ under $\sigma: X \rightarrow E_{G} / P$. Given the holomorphic principal $P$-bundle $E_{\sigma}$, we consider the adjoint bundle

$$
\operatorname{ad}\left(E_{\sigma}\right)=E_{\sigma} \times{ }_{P} \mathfrak{p}
$$


and if we have a further reduction of structure group $\sigma_{L}$ of $E_{\sigma}$ to a principal $L$-bundle $E_{\sigma_{L}}$, then we can consider also

$$
\operatorname{ad}\left(E_{\sigma_{L}}\right)=E_{\sigma_{L}} \times{ }_{L} \mathfrak{l}
$$

Let $\chi: P \rightarrow \mathbb{C}^{*}$ be an antidominant character of $P$. The degree of $E_{\sigma}$, with respect to $\chi$, denoted by $\operatorname{deg}_{\chi}\left(E_{\sigma}\right)$, is the degree of the line bundle obtained by extending the structure group of $E_{\sigma}$ through $\chi$. In other words,

$$
\operatorname{deg}_{\chi}\left(E_{\sigma}\right)=\operatorname{deg}\left(E_{\sigma} \times{ }_{\chi} \mathbb{C}^{*}\right) .
$$

Here is the general definition of (semi,poly)stability of $G$-Higgs bundles over a compact Riemann surface $X$ and for $G$ a reductive complex Lie group. It depends on a parameter $\alpha \in i \mathfrak{z}_{\mathfrak{h}}$, where $\mathfrak{z}_{\mathfrak{h}}$ denotes the center of $\mathfrak{h}$. Recall that $\langle\cdot, \cdot\rangle$ denotes an invariant $\mathbb{C}$-bilinear pairing on $\mathfrak{g}$ extending the Killing form on the semisimple part $\mathfrak{g}_{s s}$. Details may be found in [14, where these conditions were defined in the more general setting of any principal pairs for any real reductive Lie group.

Definition 3.1. Let $\alpha \in i \mathfrak{z} \mathfrak{h}$. A G-Higgs bundle $\left(E_{G}, \varphi\right)$ over $X$ is:

- $\alpha$-semistable if $\operatorname{deg}_{\chi}\left(E_{\sigma}\right)-\left\langle\alpha, s_{\chi_{*}}\right\rangle \geqslant 0$, for any parabolic subgroup $P$ of $G$, any nontrivial antidominant character $\chi$ of $P$ and any reduction of structure group $\sigma$ of $E_{G}$ to $P$ such that $\varphi \in H^{0}\left(X, \operatorname{ad}\left(E_{\sigma}\right) \otimes K\right)$.

- $\alpha$-stable if $\operatorname{deg}_{\chi}\left(E_{\sigma}\right)-\left\langle\alpha, s_{\chi_{*}}\right\rangle>0$, for any parabolic subgroup $P$ of $G$, any non-trivial antidominant character $\chi$ of $P$ and any reduction of structure group $\sigma$ of $E_{G}$ to $P$ such that $\varphi \in H^{0}\left(X, \operatorname{ad}\left(E_{\sigma}\right) \otimes K\right)$.

- $\alpha$-polystable if it is $\alpha$-semistable and if the following holds. Suppose that $\operatorname{deg}_{\chi}\left(E_{\sigma}\right)-$ $\left\langle\alpha, s_{\chi_{*}}\right\rangle=0$, for some parabolic subgroup $P \subset G$, some non-trivial strictly antidominant character $\chi$ of $P$ and some reduction of structure group $\sigma$ of $E_{G}$ to $P$ such that $\varphi \in H^{0}\left(X, \operatorname{ad}\left(E_{\sigma}\right) \otimes K\right)$. Then there is a further holomorphic reduction of structure group $\sigma_{L}$ of $E_{\sigma}$ to the Levi subgroup $L$ of $P$ such that $\varphi \in H^{0}\left(X, \operatorname{ad}\left(E_{\sigma_{L}}\right) \otimes K\right)$.

Remark 3.2. A $G$-Higgs bundle with $\varphi=0$ is a holomorphic principal $G$-bundle and a (semi)stability condition for these objects over compact Riemann surfaces was established by Ramanathan in [30]. A direct generalization of Ramanathan's condition to the G-Higgs bundle case (for $G$ complex) is given in [3]. In both cases, we see that the stability does not depend on any parameter $\alpha$. There is however no discrepancy between both (semi)stability conditions, because in [30, 3] the authors only consider characters which are trivial on the center of $G$. The corresponding ones on the Lie algebra are thus orthogonal to $\alpha$ with respect to the pairing $\langle\cdot, \cdot\rangle$, hence the parameter vanishes on the conditions. The above definition of [14] is finer in the sense that it makes precise that there is a parameter involved. One can say that the precise relation between both conditions is hence that a $G$-Higgs bundle $\left(E_{G}, \varphi\right)$ is (semi)stable in the sense of [30, 3] if and only if it is $\alpha$-(semi)stable in the sense of [14, for some $\alpha$. The significance of the parameter $\alpha$ is more obvious in the generalization of the notions of Higgs bundle and stability for real reductive Lie groups (see [14]). When $G$ is complex, given a $G$-Higgs bundle $\left(E_{G}, \varphi\right)$, we shall see in fact below (cf. Proposition 3.4) that the value of the parameter $\alpha$ is uniquely determined by the topological type of $\left(E_{G}, \varphi\right)$. In other words, $\left(E_{G}, \varphi\right)$ can only be $\alpha$-polystable if $\alpha$ is the element in $i_{\mathfrak{z} \mathfrak{h}}$ determined by the topological type of $E_{G}$.

Denote by $\mathcal{M}_{G}^{\alpha}(c)$ the moduli space of $\alpha$-semistable $G$-Higgs bundles with fixed topological class $c$ over the Riemann surface $X$. As usual, the moduli space $\mathcal{M}_{G}^{\alpha}(c)$ can also be viewed as parametrizing isomorphism classes of $\alpha$-polystable $G$-Higgs bundles. The moduli space $\mathcal{M}_{G}^{\alpha}(c)$ has the structure of a quasi-projective variety, as one can see from the Schmitt's 
general Geometric Invariant Theory construction of moduli of decorated bundles (cf. [33]), which applies in particular to the case of $G$-Higgs bundles, without assuming the connectedness of $G$ (cf. [33, Remark 2.7.5.4]). For related constructions also for higher dimensional projective varieties one can look at the work of Simpson [36, 37]

3.2. Hitchin equations and $\alpha$-polystability condition. Let $\left(E_{G}, \varphi\right)$ be a $G$-Higgs bundle over $X$. By an abuse of notation, we shall denote the $C^{\infty}$-objects underlying $E_{G}$ and $\varphi$ by the same symbols. Then the Higgs field may be viewed as a $(1,0)$-form on $X$ with values in $\operatorname{ad}\left(E_{G}\right), \varphi \in \Omega^{1,0}\left(X, \operatorname{ad}\left(E_{G}\right)\right)$. Let $H \subset G$ be a maximal compact subgroup. Then its Lie algebra $\mathfrak{h}$ is a compact form of $\mathfrak{g}$. Given a $C^{\infty}$ reduction of structure group $h$ of $E_{G}$ to $H$, let $F_{h}$ be the curvature of the corresponding Chern connection (the unique $H$-connection compatible with the holomorphic structure of $\left.E_{G}\right)$. Let also $\tau_{h}: \Omega^{1,0}\left(X, \operatorname{ad}\left(E_{G}\right)\right) \rightarrow \Omega^{0,1}\left(X, \operatorname{ad}\left(E_{G}\right)\right)$ be the involution given by the combination of complex conjugation on complex 1-forms with the compact conjugation on $\mathfrak{g}^{\mathbb{C}}$ which determines the compact form $\mathfrak{h}$, and which is given fibrewise by the metric $h$. Let $\omega$ be a volume form of $X$. The Hitchin-Kobayashi correspondence asserts the following.

Theorem 3.3. A G-Higgs bundle $\left(E_{G}, \varphi\right)$ is $\alpha$-polystable if and only if there is a reduction of structure group $h$ of $E_{G}$ from $G$ to $H$ that satisfies the Hitchin equation $F_{h}-\left[\varphi, \tau_{h}(\varphi)\right]=-i \alpha \omega$.

A proof of this correspondence can be found in [14, Theorems 2.24 and 3.21] (see also [11]), in fact in a much more general setting than the one we are considering here. Indeed, this correspondence was first proved for $G=\mathrm{SL}(2, \mathbb{C})$ by Hitchin in $[22$.

The polystability condition for a $G$-Higgs bundle $\left(E_{G}, \varphi\right)$ depends, in principle, of a parameter $\alpha \in i_{\mathfrak{z} \mathfrak{h}}$, but as $G$ is complex, $\alpha$ is indeed fixed by the topological type of $E_{G}$, as we now show.

Proposition 3.4. Let $\left(E_{G}, \varphi\right)$ be a G-Higgs bundle of topological type c. Then there is a unique value of $\alpha \in i_{\mathfrak{z} \mathfrak{h}}$, determined by $c$, for which $\left(E_{G}, \varphi\right)$ can be $\alpha$-polystable.

Proof. This can be seen combining Chern-Weil theory and Theorem 3.3. Indeed, if $\left(E_{G}, \varphi\right)$ is $\alpha$-polystable then it corresponds to a solution $h$ of $F_{h}-\left[\varphi, \tau_{h}(\varphi)\right]=-i \alpha \omega$. By applying any degree one $H$-invariant polynomial $p$ on $\mathfrak{h}$ to the equation and integrating over $X$, we obtain

$$
p(\alpha)=\frac{i}{\operatorname{vol}(X)} \int_{X}\left[p\left(F_{h}\right)\right]
$$

Notice that we have used here that $G$ is a complex group because in this case $\left[\varphi, \tau_{h}(\varphi)\right]$ is in the semisimple part of $\mathfrak{h}$, hence any $p$ vanishes on it. Chern-Weil theory implies that the cohomology class $\left[p\left(F_{h}\right)\right] \in H^{2}(X, \mathbb{C})$ only depends on the topological class $c$ of $E_{G}$. Since a degree one $H$-invariant polynomial of $\mathfrak{h}$ is a linear map $p: \mathfrak{h} \rightarrow \mathbb{C}$ which factors through $\mathfrak{h} /[\mathfrak{h}, \mathfrak{h}] \cong \mathfrak{z}_{\mathfrak{h}}$, the space of such polynomials is identified with the dual of $\mathfrak{z} \mathfrak{h}$. As $\alpha \in i \mathfrak{z} \mathfrak{h}$, then applying (3.4) simultaneously for a basis of $\mathfrak{z}_{\mathfrak{h}}^{*}$ determines $\alpha$, as required.

For example, as mentioned above, a $\operatorname{GL}(n, \mathbb{C})$-Higgs bundle is equivalent to a pair $(V, \varphi)$ where $V$ is a rank $n$ holomorphic vector bundle and $\varphi \in H^{0}(\operatorname{End}(V) \otimes K)$. The topological type of $(V, \varphi)$ is given by the degree $d$ of $V$. If we normalize the volume of $X$ to be $\operatorname{vol}(X)=2 \pi$ and take the trace as a base of the space of degree one invariant polynomials in $\mathfrak{u}(n)$, then $(V, \varphi)$ can only be $\alpha$-(semi,poly)stable in the sense of Definition 3.1 if $\alpha$ equals the slope of $V$, i.e., $\alpha=d / n$. In this case, then one checks that indeed the $d / n$-(semi,poly)stability condition is equivalent to the usual (semi,poly)stability condition comparing the slopes of $V$ and of its $\varphi$-invariants subbundles. 
Fixing the topological type $c$ thus fixes $\alpha$, and hence we can just write $\mathcal{M}_{G}(c)$ for the moduli space of polystable $G$-Higgs bundles over $X$, of topological type $c$, where it is implicit that we are using the $\alpha$ given by $c$.

Let

$$
\mathcal{M}_{G}=\bigsqcup_{c} \mathcal{M}_{G}(c),
$$

where $c$ runs over all possible topological types of $G$-Higgs bundles, according to Theorem 2.2 (if $\pi_{0} G$ abelian).

3.3. Non-emptiness. We now want to prove that the spaces $\mathcal{M}_{G}(c)$ are non-empty. For that it is enough to prove the existence, for any $c$, of a polystable $G$-principal bundle with topological type $c$ (so a $G$-Higgs bundle with vanishing Higgs field). This is well-known to be true in case $G$ is connected (see the first part of the proof of Theorem 3.6 below), so the main purpose is to prove non-emptiness of $\mathcal{M}_{G}(c)$ for non-connected $G$.

Let $H \subset G$ be a maximal compact subgroup. Let $E_{H}$ be a $C^{\infty} H$-principal bundle over $X$, with projection map

$$
p_{X}: E_{H} \rightarrow X .
$$

Let $H_{0}$ be the connected component of the identity of $H$, so that we have

$$
1 \rightarrow H_{0} \rightarrow H \stackrel{\pi}{\rightarrow} \pi_{0} H \rightarrow 1 \text {. }
$$

Let $Y$ be the quotient $E_{H} / H_{0}$. Then the projection $E_{H} \rightarrow E_{H} / H_{0}$ is a $C^{\infty} H_{0}$-principal bundle over $Y$. Denote this $H_{0}$-bundle by

$$
p_{Y}: E_{H_{0}} \rightarrow Y \text {. }
$$

Clearly the total spaces of $E_{H}$ and of $E_{H_{0}}$ are the same; they just project to different basis, having hence different fibres and structure groups. Notice also that $p: Y \rightarrow X$ is an unramified covering. It is in fact a $\pi_{0} H$-principal bundle over $X$, and $p_{X}=p \circ p_{Y}$.

Proposition 3.5. Any connection $A_{0}$ on $E_{H_{0}} \rightarrow Y$ induces naturally a connection $A$ on $E_{H} \rightarrow X$.

Proof. Let $A_{0}$ be a connection on $E_{H_{0}}$. Then $A_{0} \in \Omega^{1}\left(E_{H_{0}}, \mathfrak{h}\right)$ is an $\mathfrak{h}$-valued 1-form on $E_{H_{0}}$, such that for every $x \in E_{H_{0}}, A_{0, x}: T_{x} E_{H_{0}} \rightarrow \mathfrak{h}$ is a splitting of the exact sequence

$$
0 \rightarrow \mathfrak{h} \stackrel{v_{x, *}^{0}}{\longrightarrow} T_{x} E_{H_{0}} \stackrel{\left(p_{Y, *}\right)_{x}}{\longrightarrow} T_{p_{Y}(x)} Y \rightarrow 0
$$

where $v_{x, *}^{0}$ is the differential at the identity of the map $v_{x}^{0}: H_{0} \rightarrow E_{H_{0}}, h \mapsto x \cdot h$, given by the right $H_{0}$-action on $E_{H_{0}}$. Moreover, if $H_{x}=\operatorname{ker} A_{0, x} \subset T_{x} E_{H_{0}}$ is the horizontal subspace of $T_{x} E_{H_{0}}$, then $\left(R_{h}\right)_{*, x}\left(H_{x}\right)=H_{x \cdot h}$ for any $h \in H_{0}$, where $R_{h}: E_{H_{0}} \rightarrow E_{H_{0}}, x \mapsto x \cdot h$. Recall that $H_{x}$ is isomorphic to $T_{p_{Y}(x)} Y$ via $\left(p_{Y, *}\right)_{x}$.

Since the total spaces $E_{H_{0}}$ and $E_{H}$ are the same, we can define $A \in \Omega^{1}\left(E_{H}, \mathfrak{h}\right)$ as $A=A_{0}$. We have to see that $A$ is indeed a connection on $E_{H}$.

For every $x \in E_{H}$, it is clear that $A_{x}: T_{x} E_{H} \rightarrow \mathfrak{h}$ is a splitting of

$$
0 \rightarrow \mathfrak{h} \stackrel{v_{x, *}}{\longrightarrow} T_{x} E_{H} \stackrel{\left(p_{X, *}\right)_{x}}{\longrightarrow} T_{p_{X}(x)} X \rightarrow 0,
$$

where $v_{x, *}$ is the differential at the identity of $v_{x}: H \rightarrow E_{H}, h \mapsto x \cdot h$. Note that this restricts to the given $H_{0}$-action on $E_{H_{0}}$, so $v_{x, *}=v_{x, *}^{0}$. Note also that

$$
T_{p_{X}(x)} X \cong T_{p_{Y}(x)} Y,
$$

for every $x \in E_{H}$, since $Y \rightarrow X$ is an unramified covering. 
Pick any $x \in E_{H}$ and any $h \in H$. Let $H_{x}=\operatorname{ker} A_{x}$. We want to see $\left(R_{h}\right)_{*, x}\left(H_{x}\right)=H_{x \cdot h}$. Clearly, the right $H$-action on $E_{H}$ is equivariant with the right $\pi_{0} H$-action on $Y$, so we have the following commutative diagram, where we are implicitly using (3.8)

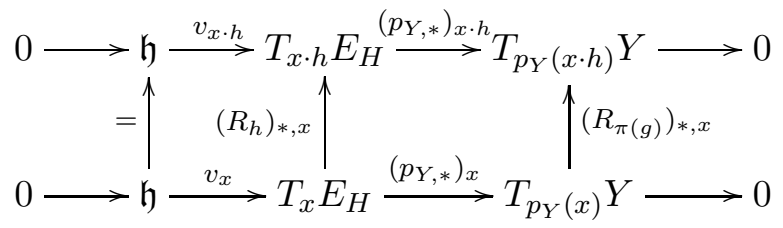

and where $\pi(h)$ is the projection of $h \in H$ in $\pi_{0} H$ as in (3.6). Since $\left(p_{Y, *}\right)_{x}$ and $\left(p_{Y, *}\right)_{x \cdot h}$ map $H_{x}$ and $H_{x \cdot h}$ respectively onto $T_{p_{Y}(x)} Y$ and $T_{p_{Y}(x \cdot h)} Y$, respectively, we conclude that indeed $\left(R_{h}\right)_{*, x}\left(H_{x}\right)=H_{x \cdot h}$.

Hence, $A$ is a connection on $E_{H} \rightarrow X$.

Theorem 3.6. For any topological type $c$ given by Theorem 2.2, there exists a polystable G-principal bundle.

Proof. Take $H$ as above. If $G$ is connected (then so is $H$ ), from Proposition 6.16 of [2] it follows that given a $C^{\infty} H$-principal bundle $E_{H} \rightarrow X$ with any topological type $c \in$ $\pi_{1} G$, it admits a Hermitian-Einstein connection (that is a connection $A$ whose curvature is constant and defined by an element in $\mathfrak{z} \mathfrak{h}$, the centre of the Lie algebra of $H$ ). From [32], that implies the polystability of the holomorphic $G$-principal bundle $E_{G}$ associated to $E_{H}$ and with the holomorphic structure such that $A$ is the Chern connection on $E_{G}$ (so the holomorphic structure on $E_{G}$ is given by $\bar{\partial}_{A}=A^{0,1}$, the $(0,1)$-part of $A$ ).

Assume that $G$ (hence $H$ ) is not connected. Let $H_{0}$ be the component of the identity. Take an $H$-principal bundle $E_{H} \rightarrow X$ in the $C^{\infty}$ category, with any topological type $c$ (given by Theorem 2.2). From it, construct the $C^{\infty} H_{0}$-principal bundle $E_{H_{0}} \rightarrow Y=E_{H} / H_{0}$ as in (3.7). Since $H_{0}$ is connected, $E_{H_{0}}$ admits a Hermitian-Einstein connection $A_{0} \in \Omega^{1}\left(E_{H_{0}}, \mathfrak{h}\right)$ such that the holomorphic $G_{0}$-principal bundle $E_{G_{0}} \rightarrow Y$ associated to $E_{H_{0}}$ and to $\bar{\partial}_{A_{0}}$ is polystable. From the preceding proposition, $A_{0}$ yields naturally a connection $A$ on $E_{H}$. Take the corresponding holomorphic $G$-principal bundle $E_{G} \rightarrow X$, coming from $\bar{\partial}_{A}$. The topological type of $E_{G}$ is still the given $c$. Finally, since $E_{G_{0}}$ is polystable, then from [4], so is $E_{G}$.

Corollary 3.7. For any topological type c given by Theorem [2.2, $\mathcal{M}_{G}(c)$ is non-empty.

3.4. Deformation theory. We briefly recall the deformation theory of $G$-Higgs bundles and, in particular, the identification of the tangent space of $\mathcal{M}_{G}$ at the smooth points. Details can be found for instance in [14, 10].

Definition 3.8. Let $\left(E_{G}, \varphi\right)$ a G-Higgs bundle over $X$. The deformation complex of $\left(E_{G}, \varphi\right)$ is the complex of sheaves on $X$ given by

$$
C^{\bullet}\left(E_{G}, \varphi\right): \operatorname{ad}\left(E_{G}\right) \stackrel{\operatorname{ad}(\varphi)}{\longrightarrow} \operatorname{ad}\left(E_{G}\right) \otimes K .
$$

Proposition 3.9. Let $\left(E_{G}, \varphi\right)$ be a G-Higgs bundle over $X$.

(i) The infinitesimal deformation space of $\left(E_{G}, \varphi\right)$ is isomorphic to the first hypercohomology group $\mathbb{H}^{1}\left(C^{\bullet}\left(E_{G}, \varphi\right)\right)$ of the complex $C^{\bullet}\left(E_{G}, \varphi\right)$;

(ii) There is a long exact sequence

$$
\begin{aligned}
0 & \longrightarrow \mathbb{H}^{0}\left(C^{\bullet}\left(E_{G}, \varphi\right)\right) \longrightarrow H^{0}\left(\operatorname{ad}\left(E_{G}\right)\right) \longrightarrow H^{0}\left(\operatorname{ad}\left(E_{G}\right) \otimes K\right) \longrightarrow \\
& \longrightarrow \mathbb{H}^{1}\left(C^{\bullet}\left(E_{G}, \varphi\right)\right) \longrightarrow H^{1}\left(\operatorname{ad}\left(E_{G}\right)\right) \longrightarrow H^{1}\left(\operatorname{ad}\left(E_{G}\right) \otimes K\right) \longrightarrow \\
& \longrightarrow \mathbb{H}^{2}\left(C^{\bullet}\left(E_{G}, \varphi\right)\right) \longrightarrow 0
\end{aligned}
$$


where the maps $H^{i}\left(\operatorname{ad}\left(E_{G}\right)\right) \rightarrow H^{i}\left(\operatorname{ad}\left(E_{G}\right) \otimes K\right)$ are induced by $\operatorname{ad}(\varphi)$.

In particular, it follows from (i) of the proposition that if $\left(E_{G}, \varphi\right)$ represents a smooth point of the moduli space $\mathcal{M}_{G}(c)$, then $\mathbb{H}^{1}\left(C^{\bullet}\left(E_{G}, \varphi\right)\right)$ is canonically isomorphic to the tangent space at this point. From this one has that

$$
\begin{aligned}
\operatorname{dim} \mathbb{H}^{1}\left(C^{\bullet}\left(E_{G}, \varphi\right)\right) & =\chi\left(\operatorname{ad}\left(E_{G}\right) \otimes K\right)-\chi\left(\operatorname{ad}\left(E_{G}\right)\right)+ \\
& +\operatorname{dim} \mathbb{H}^{0}\left(C^{\bullet}\left(E_{G}, \varphi\right)\right)+\operatorname{dim} \mathbb{H}^{2}\left(C^{\bullet}\left(E_{G}, \varphi\right)\right)
\end{aligned}
$$

where $\chi=\operatorname{dim} H^{0}-\operatorname{dim} H^{1}$ denotes the Euler characteristic.

Let $\operatorname{Aut}\left(E_{G}, \varphi\right)$ be the group of automorphisms of $\left(E_{G}, \varphi\right)$.

$$
\operatorname{Aut}\left(E_{G}, \varphi\right)=\left\{s \in \operatorname{Aut}\left(E_{G}\right) \mid \operatorname{Ad}(s)(\varphi)=\varphi\right\}
$$

where we recall that $\operatorname{Aut}\left(E_{G}\right)=H^{0}\left(\operatorname{Ad}\left(E_{G}\right)\right)$ and $\operatorname{Ad}\left(E_{G}\right)=E_{G} \times_{G} G$ with $G$ acting by conjugation. Let also aut $\left(E_{G}, \varphi\right)$ be the space of infinitesimal automorphisms of $\left(E_{G}, \varphi\right)$, defined as

$$
\left.\operatorname{aut}\left(E_{G}, \varphi\right)=\left\{s \in \operatorname{aut}\left(E_{G}\right)\right) \mid \operatorname{ad}(s)(\varphi)=0\right\},
$$

with $\operatorname{aut}\left(E_{G}\right)=H^{0}\left(\operatorname{ad}\left(E_{G}\right)\right)$ Clearly, from (ii) of Proposition 3.9, we have

$$
\mathbb{H}^{0}\left(C^{\bullet}\left(E_{G}, \varphi\right)\right) \cong \operatorname{aut}\left(E_{G}, \varphi\right) .
$$

Remark 3.10. Let $f \in \operatorname{Aut}\left(E_{G}\right)$, so that $f$ is a global section of $\operatorname{Ad}\left(E_{G}\right)$. Given $x \in X$, the element $f(x) \in \operatorname{Ad}\left(E_{G}\right)_{x}$ is identified with a conjugacy class of an element of $g \in G$ (via the identification of $\operatorname{Ad}\left(E_{G}\right)_{x}$ with $G$, up to an inner automorphism of $G$ ). Now, the closure of the orbit of $g$ under conjugation is independent of the point $x$. This is because the value at $g$ of the $G$-invariant polynomials determine the closure of the orbit of $g$ under conjugation. Since the coefficients of these polynomials are holomorphic functions and $X$ is compact, they are constants, so the closure of the conjugation orbit of $g$ is independent of $x \in X$. Indeed, in the cases we will deal with, an element of $f \in \operatorname{Aut}\left(E_{G}\right)$ will determine really a conjugation class $[g]$ of an element $g \in G$. This will follow because the orbits, under conjugation, of such $g \in G$ will be closed.

Let $Z(G)$ denote the centre of $G$. By Remark 3.10, and since $Z(G)$ acts trivially by conjugation, we can consider $Z(G)$ as a subgroup of $\operatorname{Aut}(E, \varphi)$. If $\mathfrak{z}$ denotes the Lie algebra of $Z(G)$ then, analogously, $\mathfrak{z}$ is a subalgebra of aut $\left(E_{G}, \varphi\right)$.

Definition 3.11. A G-Higgs bundle is simple if $\operatorname{Aut}\left(E_{G}, \varphi\right) \cong Z(G)$.

In order for $\left(E_{G}, \varphi\right)$ to represent a smooth point of the moduli space $\mathcal{M}_{G}$, $\operatorname{dim} \mathbb{H}^{0}\left(C^{\bullet}\left(E_{G}, \varphi\right)\right)$ and $\operatorname{dim} \mathbb{H}^{2}\left(C^{\bullet}\left(E_{G}, \varphi\right)\right)$ must have the minimum possible value. Indeed, Serre duality provides an isomorphism $\mathbb{H}^{2}\left(C^{\bullet}\left(E_{G}, \varphi\right)\right) \cong \mathbb{H}^{0}\left(C^{\bullet}\left(E_{G}, \varphi\right)\right)^{*}$, and we have the following result (cf. [10]):

Proposition 3.12. Let $\left(E_{G}, \varphi\right)$ be a stable and simple G-Higgs bundle. Then it represents a smooth point of the moduli space $\mathcal{M}_{G}(c)$.

If we are in the situation of the previous proposition, then the dimension of component of the moduli space containing $\left(E_{G}, \varphi\right)$ equals the dimension of $\mathbb{H}^{1}\left(C^{\bullet}\left(E_{G}, \varphi\right)\right)$ which, from (3.10), becomes $\operatorname{dim}(G)(2 g-2)+2 \operatorname{dim} Z(G)$. Notice that this is twice the dimension of the moduli space $\mathcal{N}_{G}$ of principal $G$-bundles on $X$. Indeed, by considering the cotangent bundle of $\mathcal{N}_{G}$, one is naturally lead to $G$-Higgs bundles, for $G$ complex. In fact, $\mathcal{M}_{G}$ strictly contains the cotangent bundle of $\mathcal{N}_{G}$. 
3.5. Stable and not simple $G$-Higgs bundles. Stable and non-simple $G$-Higgs bundles are represented by points of the moduli space which may be orbifold type singularities. In this section we show that such $G$-Higgs bundles always reduce to a stable and simple $G^{\prime}$-Higgs bundle for some smaller group $G^{\prime} \subset G$. To prove this, we need some preliminary results which may be of interest in their own right.

First we recall what is a reduction of structure group of a $G$-Higgs bundle. If $\left(E_{G}, \varphi\right)$ is a $G$-Higgs bundle, and $G^{\prime}$ is a reductive subgroup of $G$, then a reduction of structure group of $\left(E_{G}, \varphi\right)$ is a $G^{\prime}$-Higgs bundle $\left(E_{G^{\prime}}, \varphi^{\prime}\right)$ such that $E_{G^{\prime}} \hookrightarrow E_{G}$ is a holomorphic reduction of structure group of $E_{G}$ to the principal $G^{\prime}$-bundle $E_{G}^{\prime}$, and such that $\varphi^{\prime}$ maps to $\varphi$ under the embedding $\operatorname{ad}\left(E_{G^{\prime}}\right) \otimes K \hookrightarrow \operatorname{ad}\left(E_{G}\right) \otimes K$.

Proposition 3.13. Let $\left(E_{G}, \varphi\right)$ be a stable $G$-Higgs bundle which admits a reduction to a $G^{\prime}$-Higgs bundle for some complex reductive subgroup $G^{\prime} \subset G$. Let $\left(E_{G^{\prime}}, \varphi^{\prime}\right)$ be the $G^{\prime}$-Higgs bundle obtained by the reduction of the $G$-Higgs bundle $\left(E_{G}, \varphi\right)$. Then $\left(E_{G^{\prime}}, \varphi^{\prime}\right)$ is stable as a $G^{\prime}$-Higgs bundle.

Proof. Let $H^{\prime} \subset G^{\prime}$ be a maximal compact subgroup. Then $\mathfrak{g}^{\prime} \subset \mathfrak{g}$ and $\mathfrak{h}^{\prime} \subset \mathfrak{h}$. Let $s \in$ $i \mathfrak{h}^{\prime}$. Let $P_{s}^{\prime}$ be the parabolic subgroup of $G^{\prime}$ associated to $s$ as defined in (3.1). Since also $s \in i \mathfrak{h}$, it defines a parabolic subgroup $P_{s}$ of $G$ such that $P_{s}^{\prime} \subset P_{s}$. Now, take a reduction $\sigma^{\prime} \in H^{0}\left(E_{G^{\prime}}\left(G^{\prime} / P_{s}^{\prime}\right)\right)$ and denote by $E_{\sigma^{\prime}}$ be the corresponding $P_{s}^{\prime}$-bundle. Given $\sigma^{\prime}$ and the reduction of $E_{G}$ to $E_{G^{\prime}}$ one naturally obtains an induced reduction $\sigma \in H^{0}\left(E_{G}\left(G / P_{s}\right)\right)$ of $E_{G}$ to a principal $P_{s}$-bundle $E_{\sigma}$, by extending the structure group of $E_{\sigma^{\prime}}$ through the inclusion $P_{s}^{\prime} \hookrightarrow P_{s}$. Moreover, if $\sigma^{\prime}$ is such that $\varphi^{\prime} \in H^{0}\left(\operatorname{ad}\left(E_{\sigma^{\prime}}\right) \otimes K\right)$, then $\sigma$ is such that $\varphi \in H^{0}\left(\operatorname{ad}\left(E_{\sigma}\right) \otimes K\right)$. Also, any antidominant character $\chi^{\prime}: P_{s}^{\prime} \rightarrow \mathbb{C}^{*}$ of $P_{s}^{\prime}$ gives naturally rise to an antidominant character $\chi: P_{s} \rightarrow \mathbb{C}^{*}$ of $P_{s}$, just by extending $\chi^{\prime}$ to $P_{s}$ by 1 , and clearly $\operatorname{deg}_{\chi^{\prime}}\left(E_{\sigma^{\prime}}\right)=\operatorname{deg}_{\chi}\left(E_{\sigma}\right)$. So, from Definition 3.1 of stability, we conclude that if the $G^{\prime}$-Higgs bundle $\left(E_{G^{\prime}}, \varphi^{\prime}\right)$ is not stable, then $\left(E_{G}, \varphi\right)$ is not stable.

Proposition 3.14. Let $\left(E_{G}, \varphi\right)$ be a stable G-Higgs bundle. Then every element of the Lie group $\operatorname{Aut}\left(E_{G}, \varphi\right)$ is semisimple.

Proof. The main point is given by Proposition 2.14 of [14] which says that the stability of $\left(E_{G}, \varphi\right)$ implies that every element of the infinitesimal automorphism space $\operatorname{aut}\left(E_{G}, \varphi\right)$ is semisimple. Since aut $\left(E_{G}, \varphi\right)$ is the Lie algebra of $\operatorname{Aut}\left(E_{G}, \varphi\right)$, it follows that every element of $\operatorname{Aut}\left(E_{G}, \varphi\right)_{0}$ - the identity component of $\operatorname{Aut}\left(E_{G}, \varphi\right)$ - is also semisimple. Now, take the projection morphism onto the group of connected components $p: \operatorname{Aut}\left(E_{G}, \varphi\right) \rightarrow \pi_{0}\left(\operatorname{Aut}\left(E_{G}, \varphi\right)\right)$ and let any $g \in \operatorname{Aut}\left(E_{G}, \varphi\right)$. Then $g=g_{s} g_{u}$, where $g_{s}$ is its semisimple part and $g_{u}$ its unipotent part. Since $p$ is a morphism it preserves the semisimple and unipotent parts, and since every element of the group $\pi_{0}\left(\operatorname{Aut}\left(E_{G}, \varphi\right)\right)$ is semisimple (because it is finite), then $p\left(g_{u}\right)=0$. Hence $g_{u} \in \operatorname{Aut}\left(E_{G}, \varphi\right)_{0}$. But we already know that every element in $\operatorname{Aut}\left(E_{G}, \varphi\right)_{0}$ is semisimple, so $g_{u}=0$, hence $g=g_{s}$ is semisimple.

From this result we obtain the following corollary, where again we are identifying the elements of $\operatorname{Aut}\left(E_{G}, \varphi\right)$ with elements of the $G$, up to conjugation. Given one such element $g \in \operatorname{Aut}\left(E_{G}, \varphi\right)$, let $Z_{G}(g)$ denote the centralizer in $G$ of $g$. If we choose another representative $h g h^{-1}$ in $G(h \in G)$ for the automorphism defined by $g$ then, since $Z_{G}\left(h g h^{-1}\right) \cong h Z_{G}(g) h^{-1}$, we see that $Z_{G}(g)$ is defined up to conjugation. We have the following.

Corollary 3.15. Let $\left(E_{G}, \varphi\right)$ be a stable G-Higgs bundle and let $g \in \operatorname{Aut}\left(E_{G}, \varphi\right)$. Then $Z_{G}(g)$ is reductive. 
Proof. Proving that the complex Lie group $Z_{G}(g)$ is reductive is (by definition) equivalent to proving that it is the complexification of a compact Lie group. This centralizer is the same as the subgroup of $G$ of fixed points of the inner automorphism of $G$ given by $\operatorname{Int}(g)(h)=g h g^{-1}$ :

$$
Z_{G}(g)=\{h \in G \mid \operatorname{Int}(g)(h)=h\} .
$$

This inner automorphism defines an automorphism of $G_{0}$, so $\operatorname{Int}(g) \in \operatorname{Aut}\left(G_{0}\right)$. By Proposition 3.14, $g$ is a semisimple element of $G$, hence $\operatorname{Int}(g)$ is also a semisimple element of $\operatorname{Aut}\left(G_{0}\right)$. Let $S$ be the torus in $\operatorname{Aut}\left(G_{0}\right)$ generated by $\operatorname{Int}(g)$, so that the subgroup of $G_{0}$ fixed by $S$ (or equivalently by $\operatorname{Int}(g))$ is reductive, according to [19, Proposition 3.6, page 107]. Denote this reductive group by $Z_{G_{0}}(g)$.

Now, $Z_{G}(g)$ is a finite extension of $Z_{G_{0}}(g)$ : just consider the short exact sequence

$$
0 \rightarrow Z_{G_{0}}(g) \rightarrow Z_{G}(g) \rightarrow \pi_{0}(G) \rightarrow 0
$$

where we are taking the restriction to $Z_{G}(g)$ of the projection $G \rightarrow \pi_{0}(G)$. Let $H$ be a maximal compact subgroup of $Z_{G}(g)$ (see Theorem 14.1.3 of [13]). Then $H$ intersects all the components of $Z_{G}(g)$. So $H \cap Z_{G_{0}}(g)$ is a maximal compact subgroup of $Z_{G_{0}}(g)$, whose complexification is precisely $Z_{G_{0}}(g)$ because we know that $Z_{G_{0}}(g)$ is reductive. In particular the Lie algebra of $Z_{G_{0}}(g)$ is the complexification of the Lie algebra of $H \cap Z_{G_{0}}(g)$ which, by (3.13), is equivalent to say that the Lie algebra of $Z_{G}(g)$ is the complexification of the Lie algebra of $H$. We can thus apply Proposition 15.2.4 of [13] to conclude that $Z_{G}(g)=H^{\mathbb{C}}$.

We can now have a description of stable $G$-Higgs bundles which are not simple. Some of the arguments used in the proof of the following theorem are based on similar ones used in [5].

Theorem 3.16. Let $\left(E_{G}, \varphi\right)$ be a stable G-Higgs bundle which is not simple. Then there is a complex reductive subgroup $G^{\prime} \subset G$ and a reduction of $\left(E_{G}, \varphi\right)$ to a $G^{\prime}$-Higgs bundle, which is stable and simple.

Proof. Since $\left(E_{G}, \varphi\right)$ is not simple, there is some $f \in \operatorname{Aut}\left(E_{G}, \varphi\right)$ which does not belong to $Z(G)$. Since it is stable, then [14, Proposition 3.14] aut $\left(E_{G}, \varphi\right) \cong \mathfrak{z}$, where $\operatorname{aut}\left(E_{G}, \varphi\right)$ is defined in (3.12). Notice that if $\varphi=0$, then $E_{G}$ is stable (cf. Remark 3.2) and also aut $\left(E_{G}\right) \cong \mathfrak{z}$, by Proposition 3.2 of [30]. Thus, in any case,

$$
\operatorname{Aut}\left(E_{G}, \varphi\right)_{0} \cong Z(G)_{0},
$$

$Z(G)_{0}$ denoting the connected component of $Z(G)$ containing the identity. Moreover, since $\operatorname{Aut}\left(E_{G}, \varphi\right)$ is an algebraic group, it has finitely many connected components, hence the quotient

$$
Q=\operatorname{Aut}\left(E_{G}, \varphi\right) / Z(G)
$$

is a finite group (note that $Z(G)$ is a normal subgroup of $\operatorname{Aut}\left(E_{G}, \varphi\right)$ ). Write

$$
Q=\left\{\left[f_{1}\right], \ldots,\left[f_{k}\right]\right\}
$$

where $f_{i} \in \operatorname{Aut}\left(E_{G}, \varphi\right)$.

Consider the class $\left[f_{1}\right]$. From this class we shall obtain a reduction of structure group of $E$. Recall that $f_{1}$ is a section of $\operatorname{Ad}\left(E_{G}\right)=E_{G} \times{ }_{G} G$ and that, by Remark 3.10, the automorphism $f_{1}$ determines a closure of the orbit of an element $g_{1} \in G$, under conjugation. However, by Proposition 3.14, $f_{1}$ is semisimple, hence so is $g_{1}$. Therefore its orbit by conjugation is closed, so $f_{1}$ is identified with a conjugacy class $\left[g_{1}\right]$ of $G$. Now, let $q: E_{G} \times G \rightarrow \operatorname{Ad}\left(E_{G}\right)$ be the quotient map, and define

$$
\mathcal{I}=q^{-1}\left(f_{1}(X)\right) \subset E_{G} \times G .
$$


If $p_{G}: E_{G} \times G \rightarrow G$ and $p_{X}: E_{G} \times G \rightarrow X$ are the natural projections then, as $f_{1}(X)=\left[g_{1}\right]$, we have that

$$
\left(p_{X} \times p_{G}\right)(\mathcal{I})=X \times\left(G \cdot g_{1}\right),
$$

where $G \cdot g_{1}$ denotes the orbit of $g_{1}$ under the action of $G$ on itself by conjugation. Now, let $\widehat{\mathcal{I}} \subset \mathcal{I}$ be given as

$$
\widehat{\mathcal{I}}=\left.\left(p_{X} \times p_{G}\right)\right|_{\mathcal{I}} ^{-1}\left(X \times\left\{g_{1}\right\}\right) .
$$

Let $p_{E_{G}}: E_{G} \times G \rightarrow E_{G}$ be the projection, and define

$$
E_{1}=p_{E_{G}} \mid \mathcal{I}(\widehat{\mathcal{I}}) \subset E_{G} .
$$

The restriction to $E_{1}$ of the projection $\pi: E_{G} \rightarrow X$ is holomorphic and surjective, and the centralizer $Z_{G}\left(g_{1}\right)$ of $g_{1}$ in $G$ acts transitively on the fibres of $\left.\pi\right|_{E_{1}}$. Hence, $E_{1}$ is a subbundle of $E_{G}$ whose structure group is $Z_{G}\left(g_{1}\right)$, that is, $E_{1}$ is a reduction of structure group of $E_{G}$ to $Z_{G}\left(g_{1}\right) \subset G$.

Recall now that we have made two choices. Let us see what is the dependence of our reduction on these choices. First, we have chosen a representative $f_{1} \in \operatorname{Aut}\left(E_{G}, \varphi\right)$ of the class $\left[f_{1}\right] \in Q$, where $Q$ is given by (3.14). If $f_{1} z$ is another representative, with $z \in Z(G)$, then, as above, $f_{1} z$ will give rise to the conjugacy class $\left[g_{1} z\right]$ of $G$. However, doing the same construction as in the previous paragraph with $g_{1}$ replaced by $g_{1} z$, and noticing that $Z_{G}\left(g_{1} z\right)=$ $Z_{G}\left(g_{1}\right)$, one obtains the same reduction of structure group of $E_{G}$ to the $Z_{G}\left(g_{1}\right)$-bundle $E_{1}$ as before. On the other hand, given the class $f_{1}$, we can choose a different representative $g g_{1} g^{-1}$ $(g \in G)$ of the class $\left[g_{1}\right]$. This will give rise to a reduction of structure group $E_{1}^{\prime}$ of $E_{G}$ to $Z_{G}\left(g g_{1} g^{-1}\right) \cong g Z_{G}\left(g_{1}\right) g^{-1}$. The reduction of structure group of $E_{G}$ to $Z_{G}\left(g_{1}\right)$ is therefore well-defined, up to conjugation.

Let $\mathfrak{z}_{\mathfrak{g}}\left(g_{1}\right)$ be the Lie algebra of $Z_{G}\left(g_{1}\right)$. Then we have

$$
\mathfrak{z}_{\mathfrak{g}}\left(g_{1}\right)=\left\{v \in \mathfrak{g} \mid \operatorname{Ad}\left(g_{1}\right)(v)=v\right\}
$$

and the adjoint representation restricts to $\operatorname{Ad}_{1}: Z_{G}\left(g_{1}\right) \rightarrow \operatorname{GL}\left(\mathfrak{z}_{\mathfrak{g}}\left(g_{1}\right)\right)$. Moreover, $\varphi \in$ $H^{0}\left(\operatorname{ad}\left(E_{1}\right) \otimes K\right)$, because $g_{1}$ is an automorphism of $\left(E_{G}, \varphi\right)$ (cf. (3.11)). Write $\varphi_{1}$ for $\varphi$ in $H^{0}\left(\operatorname{ad}\left(E_{1}\right) \otimes K\right)$. Then $\left(E_{1}, \varphi_{1}\right)$ is a $Z_{G}\left(g_{1}\right)$-Higgs bundle.

Now we iterate this procedure. Since $Q$ is finite, this process will end and we obtain a reduction of $\left(E_{G}, \varphi\right)$ to a $G^{\prime}$-Higgs bundle $\left(E_{k}, \varphi_{k}\right)$, where $G^{\prime}=Z_{G}\left(g_{1}, \ldots, g_{k}\right)$.

The fact that $G^{\prime}$ is a complex reductive group follows from Corollary 3.15. The stability of $\left(E_{k}, \varphi_{k}\right)$ as a holomorphic $G^{\prime}$-Higgs bundle follows from the stability of $\left(E_{G}, \varphi\right)$ as a $G$ Higgs bundle and from Proposition [3.13. Finally, since $g_{i} \in Z\left(G^{\prime}\right)$, for every $i=1, \ldots, k$, we conclude that $\operatorname{Aut}\left(E_{k}, \varphi_{k}\right)=Z\left(G^{\prime}\right)$, thus $\left(E_{k}, \varphi_{k}\right)$ is simple as a $G^{\prime}$-Higgs bundle.

Remark 3.17. As far as we know, Theorem 3.16 is not in the literature even for principal bundles - our proof also holds in that case by considering $\varphi=0$.

3.6. Strictly polystable Higgs bundles. Also strictly polystable $G$-Higgs bundles correspond to singularities of $\mathcal{M}_{G}$, which this time may be more "serious" than those of orbifold type. However, as we now recall, for such $G$-Higgs bundles there is also a reduction of structure group such Higgs bundle is stable for the new group. Indeed, the following result is a particular case for the existence of a Jordan-Hölder reduction of semistable $G$-Higgs bundles, which is unique up to isomorphism (look at [14, 29] for two different proofs).

Proposition 3.18. A polystable G-Higgs bundle admits a reduction to a stable $G^{\prime}$-Higgs bundle, where $G^{\prime} \subset G$ is a complex reductive subgroup. 
Proof. If the $G$-Higgs bundle is stable, there is nothing to prove. Assume hence that it is strictly polystable. There is then some parabolic subgroup $P \subset G$, some antidominant character $\chi$ of $P$ and some reduction of structure group $\sigma$ of $E_{G}$ to $P$ such that $\varphi \in H^{0}\left(\operatorname{ad}\left(E_{\sigma}\right) \otimes K\right)$ such that $\operatorname{deg}_{\chi}\left(E_{\sigma}\right)=0$. Moreover, there is a further holomorphic reduction of structure group $\sigma_{L}$ of $E_{\sigma}$ to an principal $L$-bundle where $L$ is the Levi subgroup of $P$ such that $\varphi \in H^{0}\left(\operatorname{ad}\left(E_{\sigma_{L}}\right) \otimes K\right)$. The $L$-Higgs bundle $\left(E_{\sigma_{L}}, \varphi\right)$ is polystable (this is proved in the same way as the proof of Proposition [3.13). If it is stable, we are done. If not, we iterate this procedure, which will eventually end, yielding a $G^{\prime}$-Higgs bundle which must be stable.

\section{The Hitchin function And the subvarieties of LOCAl Minima}

4.1. The Hitchin proper function. In this section we develop all the machinery needed to count the number of connected components of the moduli space of $G$-Higgs bundles $\mathcal{M}_{G}$, defined in (3.5).

A first division of $\mathcal{M}_{G}$ into connected is given by the topological class of the Higgs bundles $\left(E_{G}, \varphi\right)$. So, the number of connected components of $\mathcal{M}_{G}$ is bounded below by the number of topological classes of $G$-Higgs bundles on the surface $X$, which can be obtain by Theorem 2.2. If $G$ is connected the lower bound is the cardinal of $\pi_{1} G$, which may be infinite. So, the real interest is to determine the connected components of $\mathcal{M}_{G}(c)$, for each class $c$.

Fix one such class $c$. In order to study $\pi_{0}\left(\mathcal{M}_{G}(c)\right)$, we use the method introduced by Hitchin in 22, which uses the non-negative real valued function given by the $L^{2}$-norm of the Higgs field. When $\mathcal{M}_{G}(c)$ is smooth, this function is a perfect Morse-Bott function, thus it is clearly a useful tool for the study of the topology of $\mathcal{M}_{G}(c)$. But even when $\mathcal{M}_{G}(c)$ is not smooth (which is the large majority of the cases), this function is still proper, so through the study of the connected components of the subvarieties of its local minima, one can draw conclusions about the connected components of $\mathcal{M}_{G}(c)$. This is by now a standard method, which has been used systematically to study the connected components, and other topological information, of $\mathcal{M}_{G}(c)$ for many classes of $G$ (see for example [8] and the references therein). Our aim is to perform this study from an intrinsic point of view, i.e., without specifying the group $G$.

Consider the real function $f: \mathcal{M}_{G}(c) \rightarrow \mathbb{R}$ defined as

$$
f\left(E_{G}, \varphi\right)=\|\varphi\|_{L^{2}}^{2}=\int_{X}|\varphi|^{2} \mathrm{dvol}=\int_{X} B\left(\varphi, \tau_{h}(\varphi)\right) \mathrm{dvol} .
$$

where $B$ is a non-degenerate quadratic form on $\mathfrak{g}$, extending the Killing form on $\mathfrak{g}_{s s}=[\mathfrak{g}, \mathfrak{g}]$ and $\tau_{h}$ is defined before Theorem 3.3 and which depends on the metric $h$ which provides the Hitchin-Kobayashi correspondence, that is, the one which is a solution to Hitchin equations see Theorem 3.3. This function $f$ is known as the Hitchin function. Using the Uhlenbeck weak compactness theorem, one can prove 22 that $f$ is proper and therefore attains a minimum on each closed subspace $\mathcal{M}^{\prime}(c)$ of $\mathcal{M}_{G}(c)$. The next result relates the connectedness of the subspaces of $\mathcal{M}^{\prime}(c)$ of local minima with the connectedness of $\mathcal{M}^{\prime}(c)$ itself.

Proposition 4.1. Let $\mathcal{M}^{\prime}(c) \subseteq \mathcal{M}_{G}(c)$ be a closed subspace and let $\mathcal{N}^{\prime} \subset \mathcal{M}^{\prime}(c)$ be the subspace of local minima of $f$ on $\mathcal{M}^{\prime}(c)$. If $\mathcal{N}^{\prime}$ is connected then so is $\mathcal{M}^{\prime}(c)$.

The idea is then to have a detailed description of the subspace of local minima of $f$, enough to draw conclusions about its connectedness. Since this method has been already applied for several cases (see for instance [23, 10]), we will only sketch it.

The strategy for studying the connectedness of $\mathcal{M}_{G}(c)$ implies that we resort on a separated approach for the following three disjoint locus of $\mathcal{M}_{G}(c)$ : 
(1) locus of stable and simple $G$-Higgs bundles;

(2) locus of stable but not simple $G$-Higgs bundles;

(3) locus of strictly polystable $G$-Higgs bundles.

Proposition 3.12 says that (1) is included in the smooth locus of $\mathcal{M}_{G}(c)$.

4.2. Description of the local minima. In this section we only consider stable and simple $G$-Higgs bundles. A very useful feature of the moduli space $\mathcal{M}_{G}(c)$ is that it carries a $\mathbb{C}^{*}$-action

$$
\lambda \cdot\left(E_{G}, \varphi\right)=\left(E_{G}, \lambda \varphi\right) .
$$

By considering the moduli space of gauge equivalence classes of solutions to the Hitchin equations and the restriction of the $\mathbb{C}^{*}$-action to an $S^{1}$-action, one concludes that a point of $\mathcal{M}_{G}(c)$ represented by a stable and simple $G$-Higgs bundle is a critical point of $f$ if and only if it is a fixed point of the $\mathbb{C}^{*}$-action; this is a consequence of the fact that $f$ is a moment map for the $S^{1}$-action (cf. [22]). Higgs bundles with vanishing Higgs field are obvious fixed points (and global minima of $f$ ) and the following result (see [22, 35]) provides a description of the other fixed points and, consequently, of the critical points of $f$.

Proposition 4.2. A stable and simple $G$-Higgs bundle $\left(E_{G}, \varphi\right)$ is fixed under the $\mathbb{C}^{*}$-action (4.2) if and only if there exists a semisimple element $\psi \in H^{0}\left(E_{G} \times_{H} \mathfrak{h}\right)$ such that there is a decomposition

$$
\operatorname{ad}\left(E_{G}\right)=\bigoplus_{k=-k_{M}}^{k_{M}} \operatorname{ad}\left(E_{G}\right)_{k}
$$

into eigenbundles of $\operatorname{ad}\left(E_{G}\right)$ under the adjoint action $\operatorname{ad}(\psi): \operatorname{ad}\left(E_{G}\right) \rightarrow \operatorname{ad}\left(E_{G}\right)$. Here, $k_{M}$ is positive integer and, for each $k, \operatorname{ad}\left(E_{G}\right)_{k}$ is the ik-eigenbundle. Furthermore, $\operatorname{ad}(\psi)(\varphi)=i \varphi$, so that $\varphi \in H^{0}\left(\operatorname{ad}\left(E_{G}\right)_{1} \otimes K\right)$.

If $\left(E_{G}, \varphi\right)$ is a fixed point of the $\mathbb{C}^{*}$-action, we can then consider an induced decomposition of the complex $C^{\bullet}\left(E_{G}, \varphi\right)$, defined in (3.9), as follows:

$$
C^{\bullet}\left(E_{G}, \varphi\right)=\bigoplus_{k=-k_{M}}^{k_{M}} C^{\bullet}\left(E_{G}, \varphi\right)_{k},
$$

where $C^{\bullet}\left(E_{G}, \varphi\right)_{k}$ is the subcomplex of $C^{\bullet}\left(E_{G}, \varphi\right)$ defined by

$$
C^{\bullet}\left(E_{G}, \varphi\right)_{k}: \operatorname{ad}\left(E_{G}\right)_{k} \stackrel{\operatorname{ad}(\varphi)_{k}}{\longrightarrow} \operatorname{ad}\left(E_{G}\right)_{k+1} \otimes K
$$

where we define $\operatorname{ad}(\varphi)_{k}=\left.\operatorname{ad}(\varphi)\right|_{\operatorname{ad}\left(E_{G}\right)_{k}}$. We say that $C^{\bullet}\left(E_{G}, \varphi\right)_{k}$ is the subcomplex of weight $k$. In turn, this yields a decomposition

$$
\mathbb{H}^{1}\left(C^{\bullet}\left(E_{G}, \varphi\right)\right)=\bigoplus_{k=-k_{M}}^{k_{M}} \mathbb{H}^{1}\left(C^{\bullet}\left(E_{G}, \varphi\right)_{k}\right)
$$

of the tangent space of $\mathcal{M}_{G}(c)$ at $\left(E_{G}, \varphi\right)$.

The following result is fundamental for the description of the smooth local minima of $f$, among the critical points which have just been described. It is a consequence of the fact that, for each $k$, the subspace $\mathbb{H}^{1}\left(C^{\bullet}\left(E_{G}, \varphi\right)_{k}\right)$ is the $(-k)$-eigenspace of the hessian of $f$ at $\left(E_{G}, \varphi\right)$. This is basically [9, Lemma 3.11]. Although the proof in that paper is for $\operatorname{GL}(n, \mathbb{C})$-Higgs bundles, the same argument works in the general setting of $G$-Higgs bundles: the key facts are that for a stable $G$-Higgs bundle, $\left(E_{G}, \varphi\right)$, the Higgs vector bundle $\left(\operatorname{ad}\left(E_{G}\right), \operatorname{ad}(\varphi)\right)$ is semistable, and that there is a natural ad-invariant isomorphism $\operatorname{ad}\left(E_{G}\right) \cong \operatorname{ad}\left(E_{G}\right)^{*} \operatorname{given}$ by 
the invariant pairing $B$ on $\mathfrak{g}$, extending the Killing form on $\mathfrak{g}_{s s}$ - see the definition of the Hitchin function in (4.1).

Proposition 4.3. Let $\left(E_{G}, \varphi\right) \in \mathcal{M}_{G}(c)$ be a stable and simple critical point of $f$. Then $\left(E_{G}, \varphi\right)$ is a local minimum if and only if either $\varphi=0$ or $\operatorname{ad}(\varphi)_{k}$ in (4.3) is an isomorphism for all $k \geqslant 1$.

We can now describe the stable and simple local minima of the Hitchin function $f$.

Proposition 4.4. Let $\left(E_{G}, \varphi\right)$ be a stable and simple G-Higgs bundle, which is a critical point of $f$. Then $\left(E_{G}, \varphi\right)$ represents a local minimum if and only if $\varphi=0$.

Proof. Let $\left(E_{G}, \varphi\right)$ be a local minimum of $f$ and suppose that $\varphi \neq 0$. Consider the complex (4.3). Consider the highest possible weight $k_{M}$, i.e the highest weight such that $\operatorname{ad}\left(E_{G}\right)_{k_{M}} \neq 0$. As $\varphi \neq 0$, then $k_{M} \geqslant 1$, so by Proposition 4.3 ,

$$
\operatorname{ad}\left(E_{G}\right)_{k_{M}} \cong \operatorname{ad}\left(E_{G}\right)_{k_{M}+1} \otimes K=0,
$$

a contradiction.

Now we consider $G$-Higgs bundles which are stable but not simple. In principle, these do not correspond to smooth points of the moduli space, so our analysis of the local minima of this kind must be carried out in a different way, because Proposition 4.3 does not apply. However, given their description by Theorem 3.16, we now achieve easily the goal of describing the local minima of the Hitchin function (4.1) on the stable locus of the moduli space $\mathcal{M}_{G}(c)$.

Proposition 4.5. Let $\left(E_{G}, \varphi\right)$ be a stable $G$-Higgs bundle. Then $\left(E_{G}, \varphi\right)$ represents a local minimum of the Hitchin function if and only if $\varphi=0$.

Proof. The stable and simple case is the content of Proposition 4.4. Assuming hence that $\left(E_{G}, \varphi\right)$ is not simple, Theorem [3.16 assures the existence of a complex reductive subgroup $G^{\prime} \subset G$ and a reduction of $\left(E_{G}, \varphi\right)$ to a $G^{\prime}$-Higgs bundle $\left(E_{G^{\prime}}, \varphi^{\prime}\right)$, which is stable and simple. On the corresponding moduli space $\mathcal{M}_{G^{\prime}},\left(E_{G^{\prime}}, \varphi^{\prime}\right)$ must be a local minima of the restriction of Hitchin function to $\mathcal{M}_{G^{\prime}}$. Proposition 4.4 implies that $\varphi^{\prime}=0$, thus $\varphi=0$.

The remaining case to be considered is the locus of strictly polystable $G$-Higgs bundles. From Proposition 3.18, we achieve the description of the subvariety of local minima of $f$ in $\mathcal{M}_{G}(c)$. Let $\mathcal{N}_{G}$ denote the moduli space $\mathcal{N}_{G}$ of polystable principal $G$-bundles over $X$, and let $\mathcal{N}_{G}(c)$ be the subspace of $\mathcal{N}_{G}$ given by those $G$-bundles with topological class determined by $c$.

Theorem 4.6. The subvariety of local minima of the Hitchin function over $\mathcal{M}_{G}(c)$ is isomorphic to $\mathcal{N}_{G}(c)$.

Proof. Let $\left(E_{G}, \varphi\right)$ be a polystable $G$-Higgs bundle which is a local minimum of the Hitchin function. If it is stable, Proposition 4.5 states that $\varphi$ must vanish. If it is strictly polystable then, using Proposition 3.18 and along the same lines as the proof of Proposition 4.5, one concludes that $\varphi=0$ as well. So the local minima of $f$ is the subvariety of $\mathcal{M}_{G}(c)$ given by those $\left(E_{G}, 0\right)$, which is isomorphic to $\mathcal{N}_{G}(c)$. 


\section{ConneCted COMPOnents}

In [31], Ramanathan has shown that if $G$ is a connected reductive complex Lie group, then $\mathcal{N}_{G}(c)$ is connected for topological class $c$ in $\pi_{1} G$. However his arguments readily adapt to the case where $G$ is non-connected. For the benefit of the reader we provide the details.

Proposition 5.1. For a complex reductive Lie group $G$ and any topological class $c$, the moduli space $\mathcal{N}_{G}(c)$ is connected.

Proof. Let $E_{G}^{\prime}$ and $E_{G}^{\prime \prime}$ represent two classes in $\mathcal{N}_{G}(c)$. Let $P$ be the underlying $C^{\infty}$ principal bundle, and let $\bar{\partial}_{A^{\prime}}$ and $\bar{\partial}_{A^{\prime \prime}}$ be the operators on $P$ defining, respectively, $E_{G}^{\prime}$ and $E_{G}^{\prime \prime}$ and given by $H$-connections $A^{\prime}$ and $A^{\prime \prime}$, respectively, where $H$ is a maximal compact subgroup of $G$.

Let $\mathbb{D}$ be an open disc in $\mathbb{C}$ containing 0 and 1 . Consider the $C^{\infty}$ principal $G$-bundle $\mathbb{E}_{G} \rightarrow \mathbb{D} \times X$, where $\mathbb{E}_{G}=\mathbb{D} \times P$. Define the connection form on $\mathbb{E}_{G}$ by

$$
A_{z}(v, w)=z A^{\prime \prime}(w)+(1-z) A^{\prime}(w) \in \Omega^{1}\left(\mathbb{E}_{G}, \mathfrak{g}\right)
$$

where $v$ is tangent to $\mathbb{D}$ at $z$ and $w$ is tangent to $P$ at some point $p$. If we consider the holomorphic bundle $E_{z}$ given by $\left.\mathbb{E}_{G}\right|_{\{z\} \times X}$ with the holomorphic structure given by $A_{z}$, then we have that $E_{0} \cong E_{G}^{\prime}$ and $E_{1} \cong E_{G}^{\prime \prime}$.

As semistability is an open condition with respect to the Zariski topology, $\mathbb{D} \backslash D^{\prime}$ is connected where $D^{\prime}=\left\{z \in \mathbb{D}: E_{z}\right.$ is not semistable $\}$. Hence $\left\{E_{z}\right\}_{z \in \mathbb{D} \backslash D^{\prime}}$ is a connected family of semistable principal $G$-bundles joining $E_{0}$ and $E_{1}$. Since $E_{0} \cong E_{G}^{\prime}$ and $E_{1} \cong E_{G}^{\prime \prime}$, using the universal property of the coarse moduli space $\mathcal{N}_{G}(c)$ of $G$-principal bundles, we conclude that there is a connected family in $\mathcal{N}_{G}(c)$ joining $E_{G}^{\prime}$ and $E_{G}^{\prime \prime}$.

We can now state our main result.

Theorem 5.2. Let $G$ be a complex reductive Lie group and let $c$ be a topological class of $G$-Higgs bundles. Then $\mathcal{M}_{G}(c)$ is non-empty and connected. Thus there is a bijection between $\pi_{0}\left(\mathcal{M}_{G}\right)$ and the set of topological classes of $G$-Higgs bundles which, when $\pi_{0} G$ is abelian, are given by Theorem 2.2. In particular, if $G$ is connected, the number of connected components of $\mathcal{M}_{G}$ equals the cardinal of $\pi_{1} G$.

Proof. Immediate from Corollary 3.7, Proposition 4.1, Theorem 4.6 and Proposition 5.1,

Let $\Gamma$ be the universal central extension of $\pi_{1} X$. It is the finitely generated group defined as follows:

$$
\left.\Gamma=\left\langle a_{1}, b_{1}, \ldots, a_{g}, b_{g}, \delta\right| \prod_{i=1}^{g}\left[a_{i}, b_{i}\right]=\delta \text { and } \delta \text { central }\right\rangle .
$$

Clearly one has the extension $0 \rightarrow \mathbb{Z} \rightarrow \Gamma \rightarrow \pi_{1} X \rightarrow 0$. Now, let

$$
\Gamma_{\mathbb{R}}=\mathbb{R} \times_{\mathbb{Z}} \Gamma .
$$

Then we have an extension

$$
0 \rightarrow \mathbb{R} \rightarrow \Gamma_{\mathbb{R}} \rightarrow \pi_{1} X \rightarrow 0
$$

A representation $\rho: \Gamma_{\mathbb{R}} \rightarrow G$ is a continuous homomorphism of groups. It is called central if $\rho(\mathbb{R}) \subset Z\left(G_{0}\right)$. In fact, since $\rho$ is continuous and $\mathbb{R}$ contains the unit element, we must have $\rho(\mathbb{R}) \subset Z\left(G_{0}\right)_{0}$. Notice that if $G$ is semisimple then a central representation $\rho: \Gamma_{\mathbb{R}} \rightarrow G$ is really a representation of $\pi_{1} X$ in $G$. For a central representation $\rho$, we must then have $\rho(\delta) \in Z\left(G_{0}\right)_{0}$, but since $\prod_{i=1}^{g}\left[a_{i}, b_{i}\right]=\delta$, we must in fact have $\rho(\delta) \in Z\left(G_{0}\right)_{0} \cap G_{s s}$ where $G_{s s}$ denotes the semisimple part of $G$. 
Let $\operatorname{Hom}_{\text {cent }}^{\text {red }}\left(\Gamma_{\mathbb{R}}, G\right)$ the space of reductive representations (i.e. the ones which become completely reducible when composed with Ad : $G \rightarrow \mathrm{GL}(\mathfrak{g}))$ which are central. The group $G$ acts by conjugation on this space, as $(g \cdot \rho)(\gamma)=g \rho(\gamma) g^{-1}$. The $G$-character variety of $X$ is the quotient space

$$
\mathcal{R}_{G}=\operatorname{Hom}_{\text {cent }}^{\text {red }}\left(\Gamma_{\mathbb{R}}, G\right) / G \text {. }
$$

Non-abelian Hodge theory on $X$ establishes a homeomorphism between $\mathcal{M}_{G}$ and $\mathcal{R}_{G}$ (cf. [22, 34, 35, 14]). Let $\mathcal{R}_{G}(c)$ be the subspace of $\mathcal{R}_{G}$ whose corresponding projectively flat $G$-bundle belongs to the topological class $c$. A direct consequence of Theorem 5.2 is hence the following.

Theorem 5.3. Let $G$ be a complex reductive Lie group and let $c$ be a topological class of $G$-Higgs bundles. Then $\mathcal{R}_{G}(c)$ is non-empty and connected. Thus there is a bijection between $\pi_{0}\left(\mathcal{R}_{G}\right)$ and the set of topological classes of $G$-Higgs bundles which, when $\pi_{0} G$ is abelian, are given by Theorem 2.2. In particular, if $G$ is connected, the number of connected components of $\mathcal{R}_{G}$ equals the cardinal of $\pi_{1} G$.

Remark 5.4.

(i) In the case of $G$ complex, reductive and connected, this result also follows from the work [12] of Donagi and Pantev. The methods used there are however completely different than the ones used in this paper. There the Hitchin system is the main tool and the authors prove that the generic fibre of the Hitchin map has precisely $\pi_{1} G$ connected components. From this the conclusion that $\pi_{0}\left(\mathcal{M}_{G}\right)=\pi_{1} G$ follows. On the other hand, it seems to us that our method is more suitable for considering a generalisation of this intrinsic study of $\pi_{0}\left(\mathcal{M}_{G}\right)$ for any real reductive Lie group.

(ii) If $G$ is connected and semisimple, the main result of this paper has also already been proved by $\mathrm{J}$. Li, in [25], using different methods, involving the study of flat bundles on Riemann surfaces. Our result provides thus also an alternative proof to Li's theorem in that case. Finally, very recently, Li's result has been generalised, by N-K. Ho and C-C. M. Liu, to include representations of the fundamental group of $X$ into a complex, connected and reductive Lie group - see the Appendix of [24]. Notice that they study the components of $\operatorname{Hom}\left(\pi_{1} X, G\right)$, but these are in bijection to the ones of $\operatorname{Hom}^{\text {red }}\left(\pi_{1} X, G\right)$, since, by Theorem 8 of [16], every representation from $\pi_{1} X$ to $G$ can be deformed to a reductive one. Their method is a direct approach, using representations and using also Li's theorem. However, the representations considered there do not include all the possible topological types (for that, one needs to consider representations of $\Gamma_{\mathbb{R}}$ ), so our result is a generalisation of theirs, even in the case when $G$ is connected.

\section{REFERENCES}

[1] M. Aparicio Arroyo, O. García-Prada, Higgs bundles for the Lorentz group, Illinois J. Math. 55 (2011), 1299-1326.

[2] M. F. Atiyah, R. Bott, The Yang-Mills equations over Riemann surfaces, Philos. Trans. Roy. Soc. London Ser. A 308 (1982), 523-615.

[3] I. Biswas, T. L. Gómez, Connections and Higgs fields on a principal bundle, Ann. Glob. Anal. Geom. 33 (2008), 19-46.

[4] I. Biswas, T. Gómez, Semistability of principal bundles on a Kähler manifold with a non-connected structure group, SIGMA Symmetry Integrability Geom. Methods Appl. 10 (2014), Paper 013, 7 pp.

[5] I. Biswas, A. J. Parameswaran, On the equivariant reduction of structure group of a principal bundle to a Levi subgroup, J. Math. Pures Appl. 85 (2006), 54-70.

[6] S. B. Bradlow, O. García-Prada, P. B. Gothen, Surface group representations and U $(p, q)$-Higgs bundles, J. Diff. Geom. 64 (2003), 111-170. 
[7] S. B. Bradlow, O. García-Prada, P. B. Gothen, Representations of surface groups in the general linear group, Proceedings of the XII Fall Workshop on Geometry and Physics, Coimbra, 2003, Publicaciones de la RSME, 7 (2004), 83-94.

[8] S. B. Bradlow, O. García-Prada, P. B. Gothen, Maximal surface group representations in isometry groups of classical Hermitian symmetric spaces, Geometriae Dedicata 122 (2006), 185-213.

[9] S. B. Bradlow, O. García-Prada, P. B. Gothen, Homotopy groups of moduli spaces of representations, Topology 47 (2008), 203-224.

[10] S. B. Bradlow, O. García-Prada, P. B. Gothen, Higgs bundles for the non-compact dual of the special orthogonal group, Geom. Dedicata 175 (2015), 1-48.

[11] S. B. Bradlow, O. García-Prada, I. Mundet i Riera, Relative Hitchin-Kobayashi correspondences for principal pairs, Quart. J. Math. 54 (2003), 171-208.

[12] R. Donagi, T. Pantev, Langlands duality for Hitchin systems, Invent. Math. 189 (2012), 653-735.

[13] J. Hilgert, K.-H. Neeb, Structure and Geometry of Lie Groups, Springer Monographs in Mathematics, Springer-Verlag, 2012.

[14] O. García-Prada, P. B. Gothen, I. Mundet i Riera, The Hitchin-Kobayashi correspondence, Higgs pairs and surface group representations, Preprint arXiv:0909.4487v3.

[15] O. García-Prada, P. B. Gothen, I. Mundet i Riera, Higgs bundles and surface group representations in the real symplectic group, J. Topology 6 (2013), 64-118.

[16] O. García-Prada, I. Mundet i Riera, Representations of the fundamental group of a closed oriented surface in $\operatorname{Sp}(4, \mathbb{R})$, Topology 43 (2004), 831-855.

[17] O. García-Prada, A. Oliveira, Higgs bundles for the non-compact dual of the unitary group, Illinois J. Math. 55 (2011), 1155-1181.

[18] O. García-Prada, A. Oliveira, Connectedness of the moduli of $\operatorname{Sp}(2 p, 2 q)$-Higgs bundles, Quarterly J. Math. 65 (2014), 931-956.

[19] V. V. Gorbatsevich, A. L. Onishchik, and E. B. Vinberg, Lie groups and Lie algebras III: Structure of Lie groups and Lie algebras, Eds. A. L. Onishchik, E. B. Vinberg, Springer-Verlag, 1993.

[20] P. B. Gothen, Components of spaces of representations and stable triples, Topology 40 (2001), 823-850.

[21] P. B. Gothen, A. Oliveira, Rank two quadratic pairs and surface group representations, Geom. Dedicata 161 (2012), 335-375.

[22] N. J. Hitchin, The self-duality equations on a Riemann surface, Proc. London Math. Soc. (3) 55 (1987), $59-126$.

[23] N. J. Hitchin, Lie groups and Teichmüller space, Topology 31 (1992), 449-473.

[24] S. Lawton, D. Ramras, Covering spaces of character varieties, New York J. Math. 21 (2015), 383-416.

[25] J. Li, The Space of Surface Group Representations, Manuscripta Math. 78 (1993), 223-243.

[26] B. Martin, Reductive subgroups of reductive groups in nonzero characteristic, J. Algebra 262 (2003), $265-286$.

[27] A. Oliveira, Higgs bundles, quadratic pairs and the topology of moduli spaces, Ph.D. Thesis, Departamento de Matemática Pura, Faculdade de Ciências, Universidade do Porto, 2008.

[28] A. Oliveira, Representations of surface groups in the projective general linear group, Internat. J. Math. 22 (2011), 223-279.

[29] B. G. Otero, Jordan-Hölder reductions for principal Higgs bundles on curves, J. Geom. Phys., 60 (2010), 1852-1859.

[30] A. Ramanathan, Stable principal bundles on a compact Riemann surface, Math. Ann. 213 (1975), $129-152$.

[31] A. Ramanathan, Moduli for principal bundles over algebraic curves: II, Proc. Indian Acad. Sci. (Math. Sci.) 106 (1996), 421-449.

[32] A. Ramanathan, S. Subramanian, Einstein-Hermitian connections on principal bundles and stability, J. Reine Angew. Math. 390 (1988), 21-31.

[33] A. H. W. Schmitt, Geometric Invariant Theory and Decorated Principal Bundles, Zurich Lectures in Advanced Mathematics, European Mathematical Society, 2008.

[34] C. T. Simpson, Constructing variations of Hodge structure using Yang-Mills theory and applications to uniformization, J. Amer. Math. Soc. 1 (1988), 867-918.

[35] C. T. Simpson, Higgs bundles and local systems, Inst. Hautes Études Sci. Publ. Math. 75 (1992), 5-95.

[36] C. T. Simpson, Moduli of representations of the fundamental group of a smooth projective variety I, Publ. Math., Inst. Hautes Étud. Sci. 79 (1994), 47-129.

[37] C. T. Simpson, Moduli of representations of the fundamental group of a smooth projective variety II, Publ. Math., Inst. Hautes Étud. Sci. 80 (1995), 5-79. 


\section{O. García-Prada}

Instituto de Ciencias Matemáticas, CSIC

Nicolás Cabrera, 13-15, 28049 Madrid, Spain

email: oscar.garcia-prada@icmat.es

\section{André Oliveira}

Centro de Matemática da Universidade do Porto, CMUP

Faculdade de Ciências, Universidade do Porto

Rua do Campo Alegre 687, 4169-007 Porto, Portugal

www.fc.up.pt

email: andre.oliveira@fc.up.pt

On leave from:

Departamento de Matemática, Universidade de Trás-os-Montes e Alto Douro, UTAD

Quinta dos Prados, 5000-911 Vila Real, Portugal

www.utad.pt

email: agoliv@utad.pt 